\title{
Prediction of sediment load by sediment rating curve and neural network (ANN) in El Kebir catchment, Algeria
}

\author{
Z A Boukhrissa ${ }^{1}, \mathrm{~K}_{\text {Khanchoul }}^{1, *}$, Y Le Bissonnais ${ }^{2}$ and M Tourki $^{3}$ \\ ${ }^{1}$ Laboratory of Soils and Sustainable Development, Badji Mokhtar University-Annaba, \\ P.O. Box 12, 23000 Annaba, Algeria. \\ ${ }^{2}$ Laboratoire d'étude des Interactions-Sol-Agrosystème-Hydrosystème (LISAH), \\ 2 Place Pierre Viala, 34060 Cedex 1, Montpellier, France. \\ ${ }^{3}$ Department of Hydraulics, Badji Mokhtar University-Annaba, P.O. Box 12, 23000 Annaba, Algeria. \\ ${ }^{*}$ Corresponding author. e-mail: kam.khanchoul@gmail.com
}

The annual sediment load of a river is generally determined either from direct measurements of the sediment load throughout the year or from any of the many sediment transport equations that are available today. Due to lack of a long-term sediment concentration data, sediment rating curves and flux estimation are the most widely applied. This paper has investigated the abilities of statistical models to improve the accuracy of streamflow-suspended sediment relationships in daily and annual suspended sediment estimation. In this study, a comparison was made between suspended sediment rating curves and artificial neural networks (ANNs) for the El Kebir catchment. Daily water discharge and daily suspended sediment data from the gauging station of Ain Assel, were used as inputs and targets in the models which were based on the cascade-forward and feed-forward back-propagation using Levenberg-Marquardt and Bayesian regularization algorithms. The model results have shown that the ANN models have the highest efficiency to reproduce the daily sediment load and the global annual sediment yields. Our estimation based on the available data indicated that the areas along the El Kebir River have experienced high sediment fluxes that could have obvious impacts on the sediment trapping and siltation in the Mexa reservoir.

\section{Introduction}

Sediment yield is defined as the total sediment outflow from a watershed measurable at a point of reference during a specified period of time. Sediment outflow from the watershed is induced by processes of detachment, transportation, and deposition of soil materials by rainfall and runoff (Cigizoglu 2002). Accurate estimations of the sediment volume carried by rivers are necessary to prevent problems derived from suspended sediment load in rivers, especially in relation to the loss of water storage in reservoirs and water quality. Rivers' flow and sediment load should be observed and correlation between observation results and the basin's characteristics should be determined for well planning studies on soil and water resources development (Yenigun et al. 2008).

The estimation of this sediment load must be based on available data. Whilst flow is generally measured frequently and can be considered as a continuous record, measurements of suspended sediment concentration are usually less frequent. This lack of information about the suspended sediment concentration can result in substantial errors in estimates of the total load because storms can quickly change the rate of flow of a river. If the sampling is too infrequent, these events may be

Keywords. Sediment rating curve; artificial neural networks; sediment load prediction; El Kebir catchment. 
missed, and, even with fair sampling, it is difficult to sample a representative number of events.

The choice of a method that can be used to estimate load will usually have a major bearing on estimates derived from infrequently sampled rivers. Assessments of methods for estimating loads have been carried out using data from intensively sampled rivers or using simulated data. One of these methods, widely used when and where measured are not available (Jansson 1997; Asselman 2000; Horowitz 2003; Khanchoul and Jansson 2008), are empirical models. These models are referred to rating curves and are usually based on the linear relationship between $\log$ (concentration) and $\log$ (flow). Another picture can be obtained by taking traditional sediment rating curves that result from fitting a linear relationship between $\log$ (sediment discharge) and $\log$ (water discharge) to calculate sediment load. The datasets are used to fit the model and predict the concentrations or sediment discharges, respectively, using flow data from other times. This procedure assumes that the relationship between the concentration or sediment discharge and the other variables is representative of their relationship throughout the period of interest. Otherwise, problems can arise, such as bias, if the model is extrapolated beyond the range of the data used to fit the model, as may be required if there is no measured concentrations at extreme events.

The sediment load process is a highly nonlinear and complex system. However, the empirical regressions, despite of their inability to represent successfully, the nonlinear complex system have been widely used (Wang et al. 2006). Another way to represent the complex sediment behaviour is to assume that the processes governing sediment yield are to be stochastic and thus can be described by a stochastic process and associated probability distributions. It seems necessary that nonlinear methods such as artificial neural networks (ANNs), which are suited to complex nonlinear models, be used for the analysis of real world temporal data (Cigizoglu 2004). The ANN is capable to model any arbitrarily complex nonlinear process that relates sediment load to continuous hydro-meteorological data (Wang and Traore 2009).

ANNs are based on the present understanding of the biological nervous system, though much of the biological detail is neglected. A neural network can be trained to perform a particular function by adjusting the values of the connections (weights) between elements. The ANN learns from the input data and the associated output data, which is commonly known as the generalisation ability of the ANN (Hassan 2001). In fact, neural networks are adjusted or trained, so that a defined input leads to a specific target output. There are different types of ANN, but in the present study, two types of ANNs were used. These were the Levenberg-Marquardt Function (Lm) and Bayesian regularization $(\mathrm{Br})$ networks.

The Lm backpropagation algorithm is the default training function (trainlm) because it is very fast. Thus, it is highly recommended as a firstchoice supervised algorithm, although it does require more memory than other algorithms (Adeloye and Munari 2006). Also, trainlm performs better on function fitting (nonlinear regression) problems than on pattern recognition problems. The used algorithm is a two-layer feed-forward network with sigmoid hidden neurons and linear output neurons that can fit multi-dimensional mapping problems arbitrarily well, given consistent data and enough neurons in its hidden layer. Whereas, Bayesian regularization $(\mathrm{Br})$ neural network offers several advantages for predictive modelling including: the ability to model any function without the need to predefine it; the ability to easily handle real and categorical molecular descriptors; and insensitivity to overfitting and overtraining. Trainbr is a Bayesian regularization network training function that updates the weight and bias values according to Levenberg-Marquardt optimization. It minimizes a linear combination of squared errors and weights, and also modifies this linear combination so that at the end of training the resulting network has good generalization qualities.

Neural network approaches have been applied in a number of diverse fields, including water resources. In recent years, ANN models have attracted researchers in sediment load data (Nagy et al. 2002; Tayfur 2002; Merritt et al. 2003; Cigizoglu 2004; Kisi 2004; Agarwal et al. 2005). Jain (2001) used a single ANN approach to establish sedimentdischarge relationship and found that the ANN model could perform better than the rating curve. Cigizoglu (2004) investigated the estimation and forecasting of daily total suspended sediment load with feed-forward backpropagation method using the daily or monthly sediment load and river flow data. In the river sediment loads modelling study during storm events of short duration, Rai and Mathur (2008) found the neural network as a suitable estimation tool in two catchment areas of United States of America.

The availability of few data as well as the complex nonlinear process of sediments provided an impetus to investigate the potential of using the ANNs techniques for suspended sediment concentration modelling in El Kebir River. The study has compared the performance of ANNs and classical regression in modelling the sediment load estimation using continuous daily water discharge and suspended sediment concentration data collected for the period 1975/76-1998/99. The use of conceptual model might overcome the low performance often 
met in the regression method and improve the accuracy of river's suspended sediment concentration estimates. Besides the main objective of this research, sediment estimation was essential in the El Kebir catchment to provide basic information on the problem related to the sediment silting in the Mexa reservoir.

\section{Study area}

The El Kebir catchment is located in the extreme northeast of Algeria. The El Kebir River comes from the union of three main tributaries, Bougous River, Ballauta River, and Teboul River (figure 1). The maximum stream length within the delineated El Kebir catchment, with an area of $681 \mathrm{~km}^{2}$, is $47 \mathrm{~km}$ and there are a total of $2543 \mathrm{~km}$ of watercourses within the basin. Located three kilometers upstream from the outlet of El Kebir basin, the Mexa reservoir, with a capacity of 103 million cubic meters and an area of $651 \mathrm{~km}^{2}$, was built in the end of 1999 for civil and industrial use. The assessment of the volume of sediment loads will give information on the drainage basin erosion that will serve to protect the reservoir from high suspended sediment flux deposition.

The climate over the study basin is Mediterranean, with dry summers and rainfalls concentrated in winter periods. The average annual rainfall varied between 693 (southern part of the basin) and
$775 \mathrm{~mm}$ (northern part) with an abundance rainfall occurring in the wet season from October to May, conversely to the dry season from June to September. Although sporadic rainfall events occurred almost throughout the year, the rainy season in the area normally lasted for about 3 months (November-January). Basin land cover is largely forested associated with sparse shrubs in the higher elevations, while moderate development is primarily located in the stream valleys. The frequent use of cultivation techniques such as up and down tillage triggers rill/gully erosion and mudflow processes that, in turn, can generate severe erosion forms such as large mass movements and badlands on sandy-clay slopes.

Bedrock is primarily Oligocene sandstone and clay rocks in the hills and piedmont slopes with Cretaceous marly limestone and triasic rocks to the east and southeast in both Bougous and Ballauta valleys. Unconsolidated cover varies widely throughout the basin, with less material at the higher elevations and more in the valleys. This study site has daily streamflow data at Ain Assel gauging station and climate data from three National Agency of Hydraulic Resources (ANRH) weather stations (figure 1). Daily records were available from 1975 to 1999. Corresponding to the previous period, the mean annual water discharge was equal to $6.15 \mathrm{~m}^{3} / \mathrm{s}$, and the highest monthly values of outflows were recorded from December to February which varied between 13 and $31 \mathrm{~m}^{3} / \mathrm{s}$.

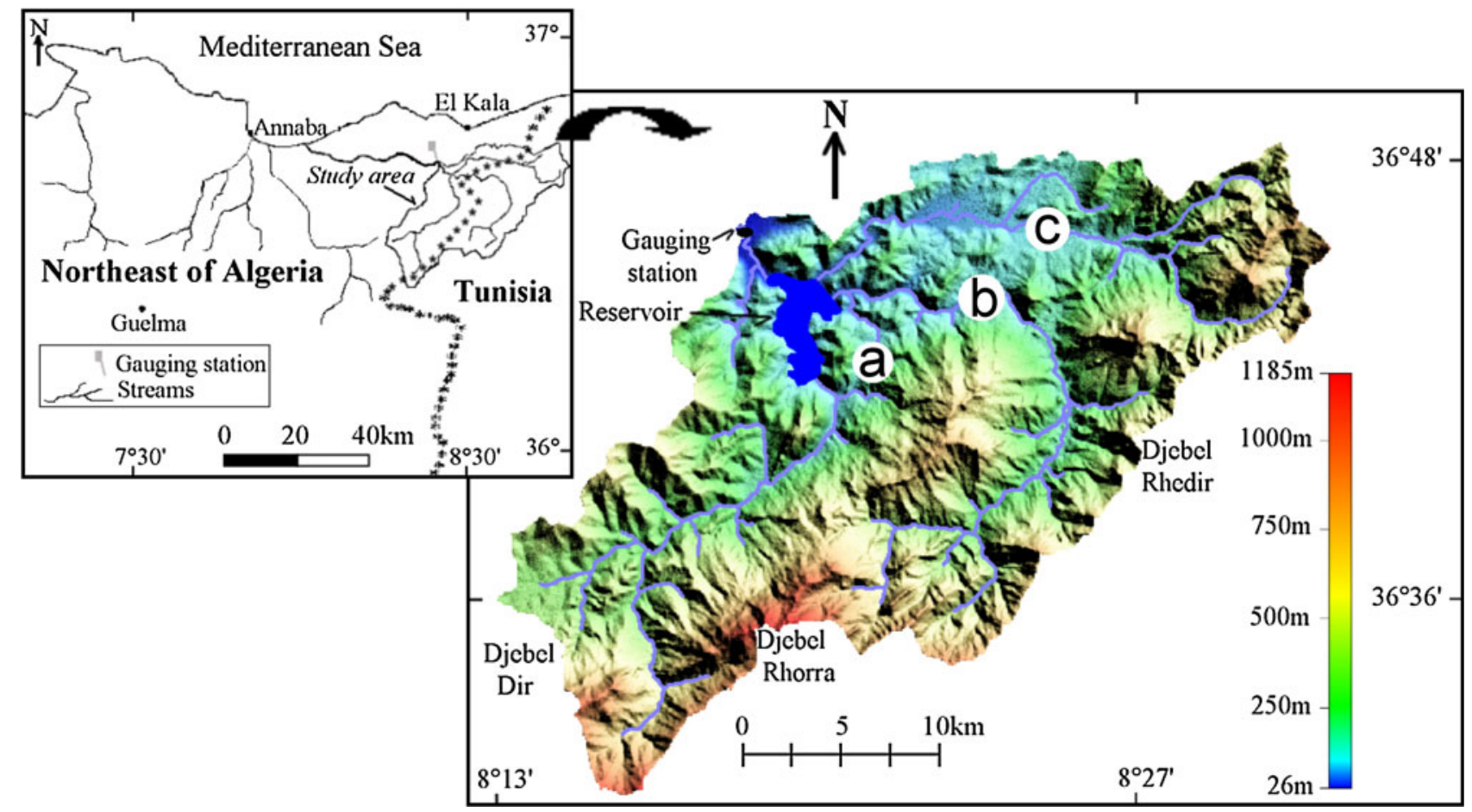

Figure 1. Location map of the El Kebir catchment and drainage network presentation. (a) Bougous River, (b) Ballauta River and (c) Teboul River. 


\section{Materials and methods}

\subsection{Suspended sediment sampling}

Fluvial data were obtained from the ANRH (National Agency of Hydraulic Resources), which operates the network of monitoring stations in Algeria. The Ain Assel hydrometric dataset included:

(1) discrete data of suspended sediment concentration $(C)$ and corresponding water discharge $(Q)$,

(2) records of daily water discharge, and

(3) hourly water discharge (based on river stage) during floods.

Daily mean water discharges were calculated as the mean of hourly water discharges.

Suspended sediment concentration samples were transported in one-litre plastic bottles for laboratory analysis. The sediment concentration of each sample was calculated by filtration method, evaporating the sample, and then weighing the remaining sediment. It was assumed that the addition of dissolved solids to the suspended sediment through evaporation was negligible. On the other hand, water samples were taken for measurement of suspended sediment concentration several times a year, which made 1086 daily datasets in 24-year period (1975-1999). Contrary to discharge of flow, the samples of sediment concentration were not available after 1999, and the collected flow datasets can be obtained from the volume of water released by the Mexa reservoir.

\subsection{Application of the sediment rating curve}

Once sufficient data have been collected, attention has been given to deriving the rating relationship. In the absence of actual suspended sediment concentration (SSC) measurements, hydrologists have used sediment rating curves to predict suspended sediment concentrations for subsequent flux calculations and to determine long-term suspended sediment loads.

In a dataset comprising 362 observations of water discharge and suspended sediment concentrations at day $(t)$, regression analyses were made between the daily suspended sediment concentration $(C)$ and the daily water discharge $(Q)$ and a relationship of the daily suspended sediment discharge $(Q s)$ versus daily water discharge $(Q)$. The most commonly used sediment rating curves are power functions (Walling 1978; Jansson 1997).

$$
\begin{gathered}
C=a Q^{b} \\
Q s=a Q^{b},
\end{gathered}
$$

where $a$ and $b$ are regression coefficients. The daily suspended sediment discharge (in $\mathrm{kg} / \mathrm{s}$ ) in the river was calculated by multiplying the daily mean sediment concentration $(\mathrm{g} / \mathrm{l})$ with the corresponding mean water discharge $\left(\mathrm{m}^{3} / \mathrm{s}\right)$.

For the regression methods presented above, we have chosen to present a further interesting prediction about the suspended sediment transport that has been extracted from the multiple regression analysis. The data considered in this regression analysis were 1086 observations. The input combinations that were tested to estimate suspended sediment values for Ain Assel station covered the daily flow at day $t\left(Q_{t}\right)$, two antecedent daily flows and daily sediment concentrations at days $t-1$ and $t-2$ $\left(Q_{t}, Q_{t-1}, Q_{t-2}, C_{t-1}, C_{t-2}\right)$. The daily sediment concentrations at day $t\left(C_{t}\right)$ were taken as outputs. For the $Q-Q s$ multiple regression, the input data of $Q_{t}, Q_{t-1}, Q_{t-2}, Q s_{t-1}, Q s_{t-2}$ at days $t, t-1$ and $t-2$ were taken as independent variables and $Q s_{t}$ at day $t$ was chosen as a dependent variable. In order to compare the performance of multiple linear regression and ANN, we divided the 1086 datasets into 978 and 924 training datasets for multiple linear regression, $108(10 \%)$ and $162(15 \%)$ sets were regression model testing for test datasets.

\subsection{Artificial neural networks and statistical performance}

The basic neural network employed in this study possessed a three-layer learning network consisting of three distinctive layers, the input layer, where the data were introduced to the ANN, the hidden layer, where data were processed, and the output layer, where the results of ANN were produced. The proposed Levenberg-Marquardt algorithm and Bayesian regularization to train the neural networks of the current research study was based on the feed-forward backpropagation method (FFBP) and cascade-forward backpropagation network $(\mathrm{CF})$. Different combinations of several internal parameters, i.e., data partitioning approach, number of hidden layers, number of neurons in each hidden layer, transfer function, error goal, etc., were tried.

The application of a function newff in relation to inputs, target, and number of neurons has created a feed-forward network. The principle of this function was to use the units where each performed a biased weighted sum of their inputs. Then, these units passed this activation level through a transfer function to produce their output, and the units were arranged in a layered feed-forward topology (figure 2a). Once the number of layers and number of units in each layer, has been selected, the network's weights and thresholds have been 
(a) Feed-forward backpropagation

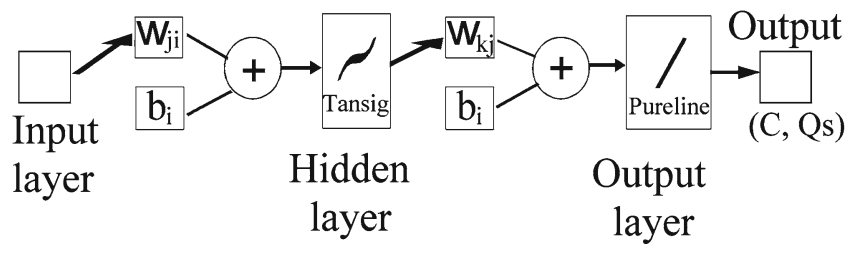

$\mathrm{W}_{\mathrm{ji}}, \mathrm{W}_{\mathrm{kj}}$ are weights and $\mathrm{b}_{\mathrm{i}}$ is the bias

(b) Cascade-forward backpropagation

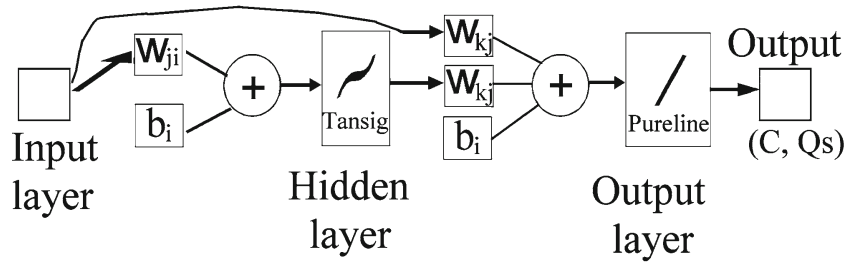

Figure 2. An exemplary three-layer feed-forward ANN structure.

set so as to minimize the prediction error made by the network. This was the role of the training algorithms. The used function 'newcf' has created cascade-forward networks. The CF included a three-layer network that has connections from layer 1 to layer 2, layer 2 to layer 3 , and layer 1 to layer 3 (figure $2 \mathrm{~b}$ ). The three-layer network also has connections from the input to all three layers. In this study, Tan-sigmoid (tansig) and pure linear (pureline) transfer functions were selected for both forward backpropagation networks to reach the optimized status.

The number of neurons in input layer varied from 1 to 3 , which represents the amount of water discharge $\left(Q_{t}\right)$ at the current day, one day $\left(Q_{t-1}\right)$ and two days $\left(Q_{t-2}\right)$ before the date of observed sediment discharge $\left(Q s_{t}\right)$ or suspended sediment concentration data $\left(C_{t}\right)$. The $Q s$ and $C$ data were taken as targets at the present time $t$ and past (antecedent) times $(t-1, t-2)$. Thereafter, the 1086 input and target data (raw data) needed to be normalized before use in the ANN training and testing to commensurate with the upper and lower bound limits of the activation functions were used in the hidden neurons. This has ensured fast processing and convergence during training and has minimized prediction error (Rojas 1996). In this study, the input and target data were preprocessed to scale the data between the range -1 and 1 using the following equation:

$$
z_{p}=2 \times \frac{\left(x_{p}-x_{\min }\right)}{\left(x_{\max }-x_{\min }\right)}-1,
$$

where $z_{p}$ is the normalized or transformed data series, $x_{p}$ is the original data series, $x_{\min }, x_{\max }$ are the minimum and the maximum values of the original data series, respectively.

The network was trained using a number of hidden neurons in the hidden layer with respect to for network with more epochs (iterations) of the neural network, which would result in different simulated results. Prior to training, the divide function was accessed automatically whenever the network was trained.

The literature offers little guidance in selecting the datasets for training and the test sample. The choice of data portions for training, testing and validation has never been subjected to general rules, where the generation of neural network models using data division has always given the users the opportunity to adjust or reduce the portions of the data used for testing and validation and of course training. In fact, most authors select the test and validation sets with the same number of data that would have an equal probability to avoid underestimation of the test or overestimation of validation and vice versa.

In this study, we have used the default method for improving generalization. This technique was automatically provided for all of the supervised network creation functions, including the backpropagation network creation functions such as newff and newcf. In this technique, the net.divideFcn (division function) was set to 'dividerand' (the default), and the available data was randomly divided into three subsets with equal proportions in the testing and validation sets. With these settings, the input vectors and target vectors were divided into three sets as follows:

- $70 \%$ and $80 \%$ were used for training.

- $15 \%$ and $10 \%$ were used to validate that the network was generalizing and to stop training before overfitting.

- The last $15 \%$ and $10 \%$ were used as a completely independent test of network generalization.

The training dataset was used to train the neural network by minimizing the error of this dataset during the training. The cross validation data were used to find the network performance by monitoring the training and guarding against overtraining. Then, the test set was used for checking the overall performance of the trained network. If the network performance was quite correct on both the test sample and the validation sample, we would be reasonably assuming that the network has a good generalization power on unknown data and less overfitting problem and therefore we would conserve the above data division; otherwise, a change in the division function could be done by taking for example 70 or $80 \%$ for training, $20 \%$ and $10 \%$ or $15 \%$ and $5 \%$ for testing and validation, respectively. 
The issue of determining the optimal number of hidden nodes is a crucial yet complicated one because there is no precise formula for selecting the optimum number of hidden neurons. In this study, the number of hidden neurons in FFBP and $\mathrm{CF}$ was decided after a series of trial runs in networks having minimum error. A rough approximation has been obtained from the Kolmogorov's Theorem. For a three-layer network, this theorem stated that twice the number of input neurons plus one is a sufficient number of hidden neurons to model any continuous function (Subramanian et al. 2004). For selecting the number of hidden neurons, we started with 2 hidden neurons and gradually increased the number until a network of 7 hidden neurons with least mean squared error was attained (Kolmogorov's application). The network was trained again by increasing the number over 7 hidden neurons. Finally, up to 12 hidden neurons, we could in some trials achieve the least mean squared error and excellent prediction of the response variable. Further increase in hidden neurons produced high error and poor network performance.

During this process, we have created a large number of networks, and we have retained more than just the best of these. Therefore, the experiments might control how many networks should be retained and the criteria were used in deciding which networks to retain.

The performance of linear regressions, feedforward backpropagation, and cascade-forward backpropagation were evaluated using Root Mean Square Error (RMSE) technique. This has been defined as:

$$
\mathrm{RMSE}=\sqrt{\frac{\sum_{i=1}^{N}\left(C_{i}-\hat{C}_{i}\right)^{2}}{N}}
$$

where $C_{i}$ is the observed sediment concentration value; $\hat{C}_{i}$ is the calculated sediment concentration value; $N$ is the number of elements; RMSE gives a quantitative indication for the model error; it measures deviation of the forecasted and/or simulated value from the actual observed value. The ideal value for RMSE is 0 .

Other possible mathematical associations were tried with the independent variables. The model efficiency factor EF of observed and predicted values were estimated for different predictions on validation datasets. The best model was selected based on the EF value approaching one. The model efficiency factor was estimated for all the validation sets using the equation:

$$
\mathrm{EF}=1-\frac{\sum_{i=1}^{n}\left(C_{i}-\hat{C}_{i}\right)^{2}}{\sum_{i=1}^{n}\left(C_{i}-\bar{C}\right)^{2}},
$$

where $n$ is the total number of observations, $C_{i}$ the $i$ th observed value, $\bar{C}$ the mean of observed values, and $\hat{C}_{i}$ the $i$ th predicted value.

Errors of estimation (E) have been calculated and expressed as percent errors of the estimated sediment load value from the observed sediment load one $\left(13.63 \times 10^{6}\right)$ as follows:

Error $(\%)=\left(\frac{\text { Estimated sediment load }}{\text { Observed sediment load }}-1\right) \times 100$.

A positive percent error represented was associated with an overestimation of the suspended sediment load and a negative percentage represented an underestimation of the value.

\section{Results and discussion}

\subsection{Sediment load-based rating curve}

The sediment rating curves developed using $Q-C$ and $Q-Q s$ for all available data from Ain Assel hydrometric station were presented in figure 3 . The
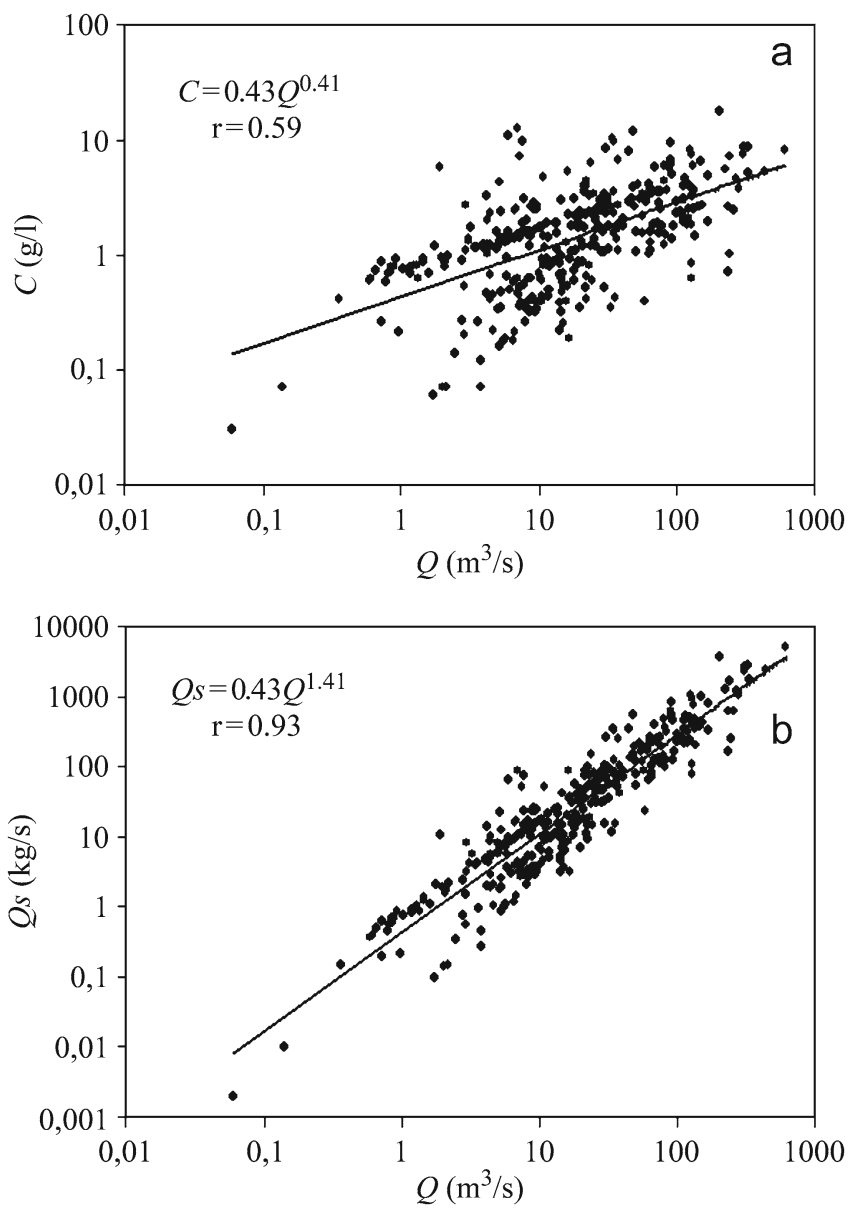

Figure 3. Relationships of (a) sediment concentration versus water discharge and (b) sediment discharge versus water discharge. 
best-fit of the theoretical functions, obtained with single derived sediment rating curve equations, have shown coefficients of correlation $(r)$ equal to 0.55 and 0.93 , respectively. The resulting RMSE and $\mathrm{EF}$ were equal to 2.00 and $0.20,233.69$ and 0.74 for the $Q-C$ and $Q-Q s$ relationships, respectively.

The best-fit power function lines through the data have underestimated the suspended sediment concentrations and suspended sediment discharges at high water discharges by $21 \%$ (table 1 ), and it is important for the high water discharges to be accurately represented because the main part of the suspended sediment load is transported during these very high discharge events. Indeed, the used sediment rating curve has given somehow a low underestimation, but the regression line can show overestimation at low water discharge values.

Based on the 90\% (978 sets) and 85\% (924 sets) training data, the training phase used to find the model parameters was deduced through the regression analysis. The multiple regression of dependent variables $C$ or $Q s$ at day $t$ versus those variables of $Q, C$, and $Q s$, at days $t, t-1, t-2$, have revealed a positive, yet somewhat a less strong relationship between water discharge and sediment concentration data but a strong relationship between water discharge and sediment discharge $(r=0.93)$. A comparative analysis, in terms of statistical measures, of RMSE, EF and $r$ is summarized in table 1. The results have shown less error and slightly better efficiency for the $Q-Q s$ relationship; however, the testing set revealed that performance of the models did not capture the complex behaviour of suspended sediments well. The results were not significant in relation to the error (RMSE > 400) and efficiency factor ( $\mathrm{EF}=0.79$ and less) values, whereas, values of $r$ in this testing phase equal to 0.91 were considered fairly good. Apparently, it seemed that the estimated suspended sediment discharges did not approximate the corresponding observed sediment discharges at medium and high values.

\subsection{Sediment load-based ANNs}

Two different types of neural networks (Lm and $\mathrm{Br}$ ) have been used to predict suspended sediments in El Kebir River. An improvement in the sediment discharge simulation performance has occurred. This improvement in the suspended sediment prediction has also been obtained through a random division of data into three subsets with equal percentages at testing and validation stages where the error in the test set did not reach a minimum at a significantly different iteration number than the validation set error; this has indicated a good division of the dataset. The amelioration was also possible through a choice of the network functions (FFBP and CF) as well as through a change of the number of neurons in the hidden layer, where they have played a significant role in ANN model performance.

The input layer combination with flows at days $t, t-1, t-2$ and $t+1$ has provided best performance criteria. After training was over, the weights were saved and used to test each network performance on test data. In both the neural network algorithms the significantly chosen number of neurons in input layer and output layer varied between 6 and 12. Generally, network training was stopped by predefined error level known as goal or on completion of predefined number of epochs (table 2). From the models listed in the later table, a possible drawback of applying the ANN approach could be done in which for each chosen data ratio division, the feed-forward or the cascade network had to be fixed during the training; meanwhile, the number of hidden neurons needed to be gradually increased for each simulation to get the best performance.

In Lm for cascade-forward network and $\mathrm{Br}$ for feed-forward backpropagation, adaptive learning procedures were followed because they have provided the best estimation of sediment discharge. From the several trainings, the ANN results have given an accepted relationship, with slight

Table 1. Statistical results of the water discharges and sediments relationships.

\begin{tabular}{|c|c|c|c|c|c|c|c|}
\hline Variables & & RMSE & $\mathrm{EF}$ & $r$ & & & \\
\hline$C-Q$ & & 2.00 & 0.20 & 0.59 & & & \\
\hline \multirow[t]{3}{*}{$Q-Q s$} & & 233.69 & 0.74 & 0.93 & & & \\
\hline & & \multicolumn{3}{|c|}{ Training } & \multicolumn{3}{|c|}{ Testing } \\
\hline & Ratio (\%) & RMSE & $\mathrm{EF}$ & $r$ & RMSE & $\mathrm{EF}$ & $r^{*}$ \\
\hline \multirow[t]{2}{*}{$C_{t}$ versus $Q_{t}, Q_{t-1}, C_{t-1}, Q_{t-2}, C_{t-2}$} & $90: 10$ & 1.80 & 0.19 & 0.61 & 2.27 & 0.37 & 0.62 \\
\hline & $85: 15$ & 1.81 & 0.17 & 0.60 & 2.08 & 0.34 & 0.62 \\
\hline \multirow[t]{2}{*}{$Q s_{t}$ versus $Q_{t}, Q_{t-1}, Q s_{t-1}, Q_{t-2}, Q s_{t-2}$} & $90: 10$ & 166.86 & 0.65 & 0.93 & 490.66 & 0.78 & 0.91 \\
\hline & $85: 15$ & 166.01 & 0.61 & 0.93 & 414.00 & 0.79 & 0.91 \\
\hline
\end{tabular}

$r^{*}$ : coefficient of correlation of the observed $C, Q s$ versus estimated $C, Q s$. 
Table 2. Performances of Lm and Br during the testing period.

\begin{tabular}{|c|c|c|c|c|c|c|c|}
\hline Hidden neurons & $\begin{array}{l}\text { Networks } \\
\text { function }\end{array}$ & $\begin{array}{l}\text { Training } \\
\text { function }\end{array}$ & RMSE & $r$ & $\mathrm{EF}$ & $\begin{array}{l}\text { SL simulated } \\
\left(\times 10^{6} \text { tonnes }\right)\end{array}$ & $\begin{array}{c}\text { Error of } \\
\text { estimation }(\%)\end{array}$ \\
\hline \multicolumn{8}{|l|}{ Ratio 80:10:10 } \\
\hline 9 & FFBP & Lm & 0.040 & 0.975 & 0.95 & 13.75 & +0.88 \\
\hline 12 & FFBP & $\mathrm{Lm}$ & 0.038 & 0.978 & 0.96 & 13.57 & -0.47 \\
\hline 9 & FFBP & $\mathrm{Br}$ & 0.040 & 0.976 & 0.95 & 13.56 & -0.51 \\
\hline 12 & FFBP & $\mathrm{Br}$ & 0.039 & 0.977 & 0.95 & 13.53 & -0.73 \\
\hline 9 & $\mathrm{CF}$ & Lm & 0.040 & 0.975 & 0.95 & 13.62 & -0.07 \\
\hline 11 & $\mathrm{CF}$ & $\mathrm{Br}$ & 0.039 & 0.977 & 0.95 & 13.58 & -0.37 \\
\hline 12 & $\mathrm{CF}$ & $\mathrm{Br}$ & 0.039 & 0.976 & 0.95 & 13.71 & +0.59 \\
\hline \multicolumn{8}{|l|}{ Ratio 70:15:15 } \\
\hline 10 & FFBP & Lm & 0.038 & 0.977 & 0.96 & 13.80 & +1.25 \\
\hline 12 & FFBP & $\mathrm{Lm}$ & 0.037 & 0.979 & 0.96 & 13.43 & -1.47 \\
\hline 6 & FFBP & $\mathrm{Br}$ & 0.040 & 0.975 & 0.95 & 13.59 & -0.29 \\
\hline 10 & FFBP & $\mathrm{Br}$ & 0.040 & 0.975 & 0.95 & 13.87 & +1.76 \\
\hline 11 & $\mathrm{CF}$ & Lm & 0.043 & 0.972 & 0.94 & 13.95 & +2.35 \\
\hline 9 & $\mathrm{CF}$ & $\mathrm{Br}$ & 0.040 & 0.975 & 0.95 & 13.49 & -1.03 \\
\hline 12 & $\mathrm{CF}$ & $\mathrm{Br}$ & 0.040 & 0.975 & 0.95 & 13.52 & -0.81 \\
\hline
\end{tabular}

Table 3. Performance evaluation of training, validation and testing for two ratio sets.

\begin{tabular}{|c|c|c|c|c|c|}
\hline Operation & Networks function & Nodes & Equation & $r$ & RMSE \\
\hline \multicolumn{6}{|c|}{ Ratio 80:10:10 } \\
\hline Training & $\mathrm{CF}(\mathrm{Lm})$ & 9 & Output $=0.94$ Target +0.059 & 0.97 & 0.116 \\
\hline Validation & $\mathrm{CF}(\mathrm{Lm})$ & 9 & Output $=0.99$ Target +0.012 & 0.99 & 0.021 \\
\hline Testing & CF (Lm) & 9 & Output $=0.77$ Target +0.220 & 0.86 & 0.439 \\
\hline All & $\mathrm{CF}(\mathrm{Lm})$ & 9 & Output $=0.95$ Target +0.046 & 0.98 & 0.094 \\
\hline \multicolumn{6}{|c|}{ Ratio $70: 15: 15$} \\
\hline Training & $\mathrm{FFBP}(\mathrm{Br})$ & 6 & Output $=0.94$ Target +0.058 & 0.97 & 0.115 \\
\hline Validation & $\mathrm{FFBP}(\mathrm{Br})$ & 6 & Output $=0.95$ Target +0.042 & 0.96 & 0.090 \\
\hline Testing & FFBP $(\mathrm{Br})$ & 6 & Output $=0.98$ Target +0.026 & 0.99 & 0.045 \\
\hline All & $\mathrm{FFBP}(\mathrm{Br})$ & 6 & Output $=0.95$ Target +0.046 & 0.98 & 0.094 \\
\hline
\end{tabular}

underestimations $(\mathrm{E}=-0.07$ in $\mathrm{CF}-\mathrm{Lm}$ with 9 nodes and $\mathrm{E}=-0.29$ in $\mathrm{FFBP}-\mathrm{Br}$ with 6 nodes). The use of a number of hidden neurons equal to 9 and 6 and a number of iterations (epochs) of 120 and 240, respectively, has provided a best validation performance of the training, validation and testing of 0.0016 and 0.24 stopped at training epochs of 60 and 200 to avoid any increase of the validation error. These performances correspond to the mean squared error (MSE) of both models. Based on the Levenberg-Marquardt and Bayesian regularization algorithms that were used for training, these relations have shown reasonable curve trend results, because the test set error and the validation set error have similar characteristics, and it did not appear that any significant overfitting has occurred.

When analyzing results from the descriptive statistics shown in table 2, the RMSE and error of estimation (\%) were found to proffer a different trend in which one parameter values were not directly proportional to the other ones. As explained previously, the error (\%) and RMSE provide different types of information about predictive capabilities of the model. Nevertheless, the reason of their dissociation was due to the fact that the RMSE measured the goodness-of-fit relevant to high values whereas the error $(\%)$ yielded a more balanced perspective of the goodness-of-fit at moderate values.

From the simulation study which was carried out on three proportions of ratio as listed in table 3 , it was found that the Bayesian regularization model has given a higher coefficient of correlation but a slightly higher RMSE during the testing; meanwhile, the validation in the $\mathrm{Lm}$ algorithm has provided a higher coefficient of correlation and a lower RMSE. The results, though producing some different patterns, often did not differ broadly by an order of magnitude between outputs and targets.

The regression performance and the computation of EF have also provided information about 
the predictive capabilities of the models. Single plot displays of network outputs with respect to targets for training, validation, and test sets were represented by a coefficient of correlation equal to 0.98 (figure 4). For a perfect fit, the data fitting a function should fall along a 45 degree line, where the network outputs are equal to the targets. On other hand, the high $r$ values resulted from the dataset of 9 and 6 neurons ANN models have provided higher values of the model efficiency factor $(\mathrm{EF}=0.95)$. Overall, the results indicated that ANNs were a promising method for predicting suspended sediment concentrations, as they have performed better than the conventional sediment rating curve method.

Using results of $Q$ and $Q s$ ANN model at days $t, t-1$, and $t-2$, it has been observed that the estimated sediment loads using CF-Lm and FFBP$\mathrm{Br}$ were equal to $22.91 \times 10^{6}$ tonnes and $23.01 \times$ $10^{6}$ tonnes respectively, corresponding to a mean annual sediment yield of $1404 \mathrm{~T} \mathrm{~km}^{-2}$ year ${ }^{-1}$ and $1410 \mathrm{~T} \mathrm{~km}^{-2}$ year $^{-1}$ during the 24-year period.
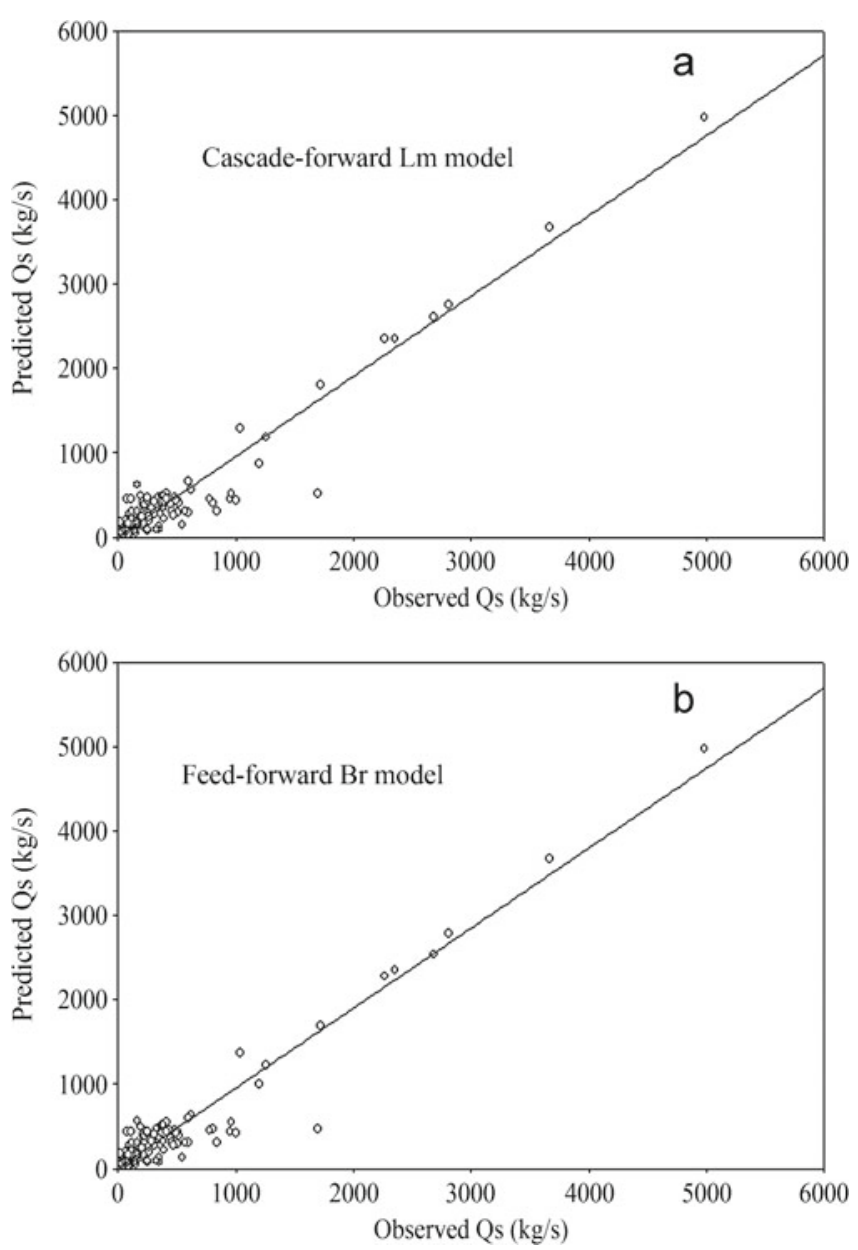

Figure 4. Observed (target) and predicted (output) suspended sediment concentrations using ANN models.
The bulk of suspended sediment transport in the El Kebir River, according to ANNs model, took place in the winter months (figure 5). The streamflows and yields were highest in the winter season (December to February) as represented by the downstream station of Ain Assel. In fact, these winter months have represented $59.60 \%(\mathrm{CF}-\mathrm{Lm})$ and $59.36 \%$ (FFBP-Br) of the total annual sediment values. Nevertheless, it appeared from figure 5 that the high sediment loads have reached their peaks before the water discharge peak. This is mainly due to more efficient sediment supply and transport during the months of December and January.

In the study catchment, sediment transport was at its lowest during the period June to August and September. Month-to-month suspended sediment concentration variability was more consistent from November to May, where the monthly means ranged from 7.00 to $3.12 \mathrm{~g} / \mathrm{l}$.

Heavy and excessive rainfall trigger high water discharge and suspended sediment load. This shows the effect of a few days with extremely high suspended sediment transport, a trend similar to water discharge in the basin (Gupta and Chakrapani 2007). A possible explanation for the considerable higher sediment yield in El Kebir River lied on the fact that morphological factors (e.g., rainfall intensity and land use) coupled with the dominant weak geological layers (e.g., clay and marl) and topography have acted as additional forces to sediment availability within the catchment. Hence, we might expect that the dam suffering from sedimentation would gradually increase in near future and consequently the rate of loss of storage capacity would increase. Lack of proper study or adequate data on reservoir sedimentation in the region was a problem in estimating the rate of silting and the economic life time of the Mexa reservoir.

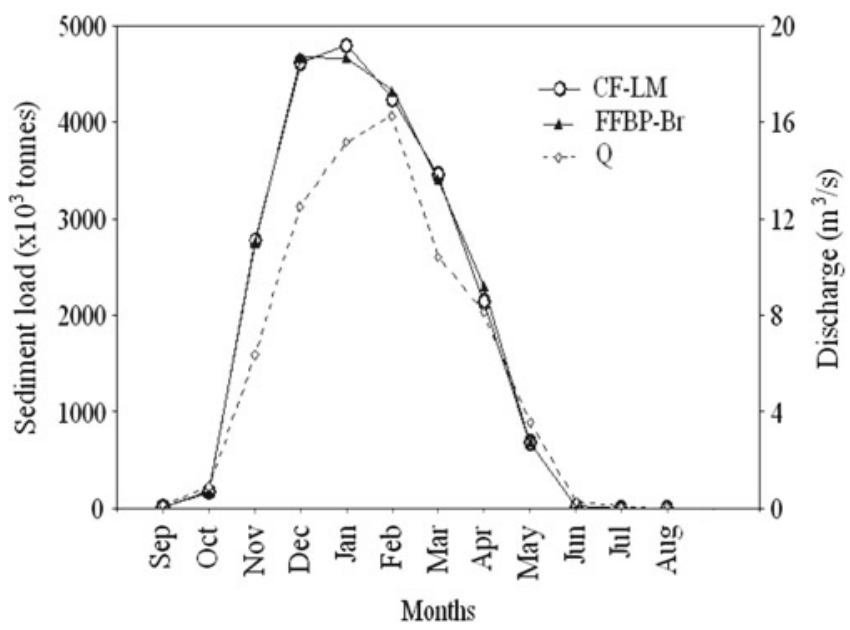

Figure 5. Seasonal pattern of suspended sediment loads in the study catchment. 


\section{Conclusion}

In the present study, an effort was made to compare the ANN and sediment rating curve models for prediction of suspended sediment load from the mean runoff resulting from the rainfall events of different intensities and durations over the $\mathrm{El}$ Kebir catchment. It was seen that including flow and suspended sediment concentration or sediment discharge in a nonlinear function could not perform a good prediction of sediment load in special conditions. Meanwhile, the use of three-layered ANN structure with the number of nodes in hidden layer via Levenberg-Marquardt and Bayesian regularization algorithms has improved the simulation results and therefore was sufficient to obtain satisfactory performance in suspended sediment load prediction. Therefore, the obtained results were of significance since the ANN predicted suspended sediment concentration values representing the average behaviour, providing a total sum close to the observed one.

Neural network can be a potential estimation method which can be used for a better understanding of sediment flux that was considered high in the study catchment. The prediction of suspended sediment loads has carried significance for the possible reservoir sedimentation in the present. Therefore, the results of this study which have shown that ANNs were an important tool in suspended sediment load simulation, could be considered as progress for sediment transport modelling.

Lack of long-term sediment load data has represented key constraints on such work, but surrogate data on past sediment obtained from the monitoring station and from reservoir sediment deposits and through space-time substitution clearly could possess considerable potential in such work.

\section{References}

Adeloye A J and Munari A D 2006 Artificial neural network based generalized storage yield-reliability models using the Levenberg-Marquardt algorithm; J. Hydrol. 362 215-230.

Agarwal A, Singh R D, Mishra S K and Bhunya P K 2005 ANN-based sediment yield models for Vamsadhara river basin (India); Water SA 31(1) 95-100.

Asselman N E M 2000 Fitting and interpretation of sediment rating curves; J. Hydrol. 234 228-248.

Cigizoglu H K 2002 Suspended sediment estimation and forecasting using artificial neural networks; Turk. J. Eng. Environ. Sci. 26 15-25.
Cigizoglu H K 2004 Estimation and forecasting of daily suspended sediment data by multilayer perceptrons; Adv. Water Resour. 27 185-195.

Gupta H and Chakrapani G J 2007 Temporal and spatial variations in water flow and sediment load in the Narmada river; Curr. Sci. 92(5) 679-684.

Hassan A E 2001 Prediction of plume migration in heterogeneous media using artificial neural networks; Water Resour. Res. 37(3) 605-623.

Horowitz A J 2003 An evaluation of sediment rating curve for estimating suspended sediment concentration for subsequent flux calculations; Hydrol. Proc. 17 3387-3409.

Jain S K 2001 Development of integrated sediment rating curves using ANNs; J. Hydraul. Eng. ASCE 127(1) 30-37.

Jansson M B 1997 Comparison of sediment rating curves developed on load and on concentration; Nordic Hydrol. 28(3) 189-200.

Khanchoul K and Jansson M B 2008 Sediment rating curves developed on stage and seasonal means in discharge classes for the Mellah wadi, Algeria; Geogr. Ann. 90A(3) $227-236$.

Kisi O 2004 Multi-layer perceptrons with LevenbergMarquardt optimization algorithm for suspended sediment concentration prediction and estimation; Hydrol. Sci. J. 49(6) 1025-1040.

Merritt W S, Letcher R A and Jakeman A J 2003 A review of erosion and sediment transport models; Environ. Modell. Softw. 18(8-9) 761-799.

Nagy H M, Watanabe K and Hirano M 2002 Prediction of sediment load concentration in rivers using artificial neural network model; J. Hydraul. Eng. 128(6) $588-595$.

Rai R K and Mathur B S 2008 Event-based sediment yield modeling using artificial neural network; Water Resour. Manag. 22(4) 423-441.

Rojas R 1996 Neural Networks: A systematic introduction (Berlin: Springer Verlag), pp. 151-184.

Subramanian N, Yajnik A and Murthy R S R 2004 Artificial neural network as an alternative to multiple regression analysis in optimizing formulation parameters of Cytarabine Liposomes; AAPS Pharm. Sci. Tech. 5(1) $1-9$.

Tayfur G 2002 Artificial neural networks for sheet sediment transport; Hydrol. Sci. J. 47(6) 879-892.

Walling D E 1978 Suspended sediment and solute response characteristics of the river Exe, Devon, England; In: Research in fluvial systems (eds) DavidsonArnott R and Nickling W, Geoabstracts (Norwich, UK), pp. 169-197.

Wang Y M and Traore S 2009 Time-lagged recurrent network for forecasting episodic event suspended sediment load in typhoon prone area; Int. J. Phys. Sci. 4(9) 519-528.

Wang Y M, Juaug S C, Lai C C and Kerh T 2006 Estimation of suspended sediment discharge for a storm; J. Univ. Sci. Technol. Beijing 28(2) 152-156.

Yenigun K, Gumus V and Bulut H 2008 Trends in streamflow of Euphrates Basin, Turkey, ICE; Water Manag. 161(4) 189-198. 